\title{
On the Variant Boussinesq Equations
}

\author{
Yi-Tian Gao and Bo Tian \\ Department of Applied Mathematics and Physics, Beijing University of Aeronautics \\ and Astronautics, Beijing 100083, China
}

Z. Naturforsch. 52 a, 335-336 (1997); received June 27, 1996

We extend the generalized tan $\mathrm{h}$ method to the variant Boussinesq equations and obtain certain solitary-wave and new exact solutions.

The Boussinesq-typed equations belong to the most famous class of nonlinear evolution equations (see e.g., [1]). The set of the variant Boussinesq equations [2],

$$
\begin{aligned}
& H_{t}+(H u)_{x}+u_{x x x}=0, \\
& u_{t}+H_{x}+u u_{x}=0,
\end{aligned}
$$

is one of the models for water waves. Lately, the set was found to possess travelling- or solitary-wave solutions with error [3] (to be explained later).

We now investigate (1) and (2) with an extended version of the generalized tanh method $[4,5]$ and symbolic computation, aiming at certain exact solutions beyond solitary waves. To begin with, we conjecture that those solutions be of the form

$$
\begin{aligned}
& u(x, t)=\mathscr{A}(x, t)+\mathscr{B}_{1}(t) \cdot \tanh [\Psi(t) x+\Theta(t)], \\
& H(x, t)=\mathscr{C}(x, t)+\mathscr{D}_{1}(t) \cdot \tanh [\Psi(t) x+\Theta(t)]+\mathscr{D}_{2}(t) \cdot \tanh ^{2}[\Psi(t) x+\Theta(t)],
\end{aligned}
$$

where $\mathscr{B}_{1}(t) \neq 0, \mathscr{D}_{2}(t) \neq 0$ and $\Psi(t) \neq 0$.

Symbolically computing (1) and (2) with (5) and (6) yields

$$
\begin{aligned}
\mathscr{B}_{1} \mathscr{C} & \Psi \operatorname{sech}^{2}(\Psi x+\Theta)+\mathscr{A} \mathscr{D}_{1} \Psi \operatorname{sech}^{2}(\Psi x+\Theta)-2 \mathscr{B}_{1} \Psi^{3} \operatorname{sech}^{4}(\Psi x+\Theta)+\mathscr{C}_{t}+\mathscr{C} \mathscr{A}_{x} \\
& +2 \mathscr{B}_{1} \mathscr{D}_{1} \Psi \operatorname{sech}^{2}(\Psi x+\Theta) \tanh (\Psi x+\Theta)+2 \mathscr{A}_{2} \mathscr{D}_{2} \Psi \operatorname{sech}^{2}(\Psi x+\Theta) \tanh (\Psi x+\Theta) \\
& +3 \mathscr{B}_{1} \mathscr{D}_{2} \Psi \operatorname{sech}^{2}(\Psi x+\Theta) \tanh ^{2}(\Psi x+\Theta)+4 \mathscr{B}_{1} \Psi^{3} \operatorname{sech}^{2}(\Psi x+\Theta) \tanh ^{2}(\Psi x+\Theta) \\
& +\mathscr{D}_{1, t} \tanh (\Psi x+\Theta)+\mathscr{D}_{2, t} \tanh ^{2}(\Psi x+\Theta)+x \mathscr{D}_{1} \Psi_{t} \operatorname{sech}^{2}(\Psi x+\Theta)+\mathscr{A}_{x}+\mathscr{A}_{x x x} \\
& +2 x \mathscr{D}_{2} \Psi_{t} \operatorname{sech}^{2}(\Psi x+\Theta) \tanh (\Psi x+\Theta)+2 \mathscr{D}_{2} \Theta_{t} \operatorname{sech}^{2}(\Psi x+\Theta) \tanh (\Psi x+\Theta) \\
& +\mathscr{D}_{1} \Theta_{t} \operatorname{sech}^{2}(\Psi x+\Theta)+\mathscr{D}_{1} \mathscr{A}_{x} \tanh (\Psi x+\Theta)+\mathscr{D}_{2} \mathscr{A}_{x} \tanh ^{2}(\Psi x+\Theta) \\
& +\mathscr{B}_{1} \mathscr{C}_{x} \tanh (\Psi x+\Theta)=0,
\end{aligned}
$$

Reprint requests and correspondence to Prof. Bo Tian. 


$$
\begin{aligned}
\mathscr{A} \mathscr{B}_{1} \Psi \operatorname{sech}^{2}(\Psi x+\Theta)+\mathscr{D}_{1} \Psi \operatorname{sech}^{2}(\Psi x+\Theta)+\mathscr{B}_{1}^{2} \Psi \operatorname{sech}^{2}(\Psi x+\Theta) \tanh (\Psi x+\Theta)+\mathscr{A} \mathscr{A}_{x} \\
\quad+2 \mathscr{D}_{2} \Psi \operatorname{sech}^{2}(\Psi x+\Theta) \tanh (\Psi x+\Theta)+\mathscr{B}_{1, t} \tanh (\Psi x+\Theta)+x \mathscr{B}_{1} \Psi_{t} \operatorname{sech}^{2}(\Psi x+\Theta) \\
\quad+\mathscr{B}_{1} \Theta_{t} \operatorname{sech}^{2}(\Psi x+\Theta)+\mathscr{A}_{t}+\mathscr{B}_{1} \mathscr{A}_{x} \tanh (\Psi x+\Theta)+\mathscr{C}_{x}=0
\end{aligned}
$$

When we equate to zero the coefficients of like powers of $\tanh (\Psi x+\Theta)$, after some algebraic manipulations (7) and (8) turn out to be

$$
\begin{array}{ll}
\tanh ^{4}(\Psi x+\Theta), \tanh ^{3}(\Psi x+\Theta): & \mathscr{D}_{2}(t)=-2 \Psi^{2}(t), \quad \mathscr{B}_{1}(t)= \pm 2 \Psi(t), \\
\tanh ^{3}(\Psi x+\Theta), \tanh ^{2}(\Psi x+\Theta): & \mathscr{A}(x, t)=\frac{ \pm \mathscr{D}_{1}(t)-\Theta_{t}(t)-\Psi_{t}(t) x}{\Psi(t)}, \quad \mathscr{D}_{1}(t)=0, \\
\tanh ^{2}(\Psi x+\Theta),-----: & \mathscr{C}=2 \Psi^{2}(t) \mp \frac{\Psi_{t}(t)}{\Psi(t)}=\mathscr{C}(t) \quad \text { only }, \\
\tanh (\Psi x+\Theta), \tanh (\Psi x+\Theta): & \text { satisfied automaticlly }
\end{array}
$$

Then, equating to zero the coefficients of like powers of $x$ with integrations gives rise to

$$
\begin{aligned}
& x^{0}, x: \quad \Psi(t)=\frac{1}{\alpha t+\beta} \quad(\beta \neq 0), \\
& -, x^{0}: \Theta(t)= \begin{cases}\frac{\gamma}{\alpha t+\beta}+\delta & \text { if } \alpha \neq 0, \\
\zeta t+\eta & \text { otherwise, }\end{cases}
\end{aligned}
$$

where $\alpha, \beta, \gamma, \delta, \zeta$ and $\eta$ are integration constants.

The last step is to substitute everything back into (5) and (6), so that we obtain a couple of families of analytical solutions for (1) and (2), as follows:

Family I: Solitary-wave solutions with $\alpha=0$

$$
\begin{aligned}
& u^{I}(x, t)= \pm \frac{2}{\beta} \tanh \left(\frac{x}{\beta}+\zeta t+\eta\right)-\beta \zeta, \\
& H^{I}(x, t)=\frac{2}{\beta^{2}} \operatorname{sech}^{2}\left(\frac{x}{\beta}+\zeta t+\eta\right) .
\end{aligned}
$$

[1] D. Zwillinger, Handbook of Differential Equations, Academic Press, Boston 1989.

[2] R. Sachs, Physica D 30, 1 (1988).

[3] M. Wang, Phys. Lett. A 199, 169 (1995).
It is easy to show that the solutions found in [3] are special cases of (15) and (16) with the choice of a " + " sign in (15). It is also noted that (25) in [3] is incorrect.

Family II: New exact solutions with $\alpha \neq 0$ and $|x| \neq \infty$

$u^{I I}(x, t)=\frac{1}{\alpha t+\beta}\left[ \pm 2 \cdot \tanh \left(\frac{x+\gamma}{\alpha t+\beta}+\delta\right)+\alpha(x+\gamma)\right]$,

$H^{I I}(x, t)=\frac{2}{(\alpha t+\beta)^{2}} \operatorname{sech}^{2}\left(\frac{x+\gamma}{\alpha t+\beta}+\delta\right) \pm \frac{\alpha}{\alpha t+\beta}$.

Those families provide information on the motion of water waves.

\section{Acknowledgements}

This work is supported by the Outstanding Young Faculty Fellowship and the Research Grants for the Scholars Returning from Abroad, State Education Commission of China. B. Tian has written the text.

[4] B. Tian and Y. T. Gao, Mod. Phys. Lett. A 10, 2937 (1995).

[5] B. Tian and Y. T. Gao, Computer Phys. Comm. 95, 139 (1996). 